\title{
REACTIONS OF THE MOTOR SYSTEM IN LOCAL AND GLOBAL ACTIVITIES
}

\author{
N. Dimitrova* \\ Department of Foreign Languages and Information Technologies, National Sports Academy \\ "Vassil Levski", Sofia, Bulgaria
}

\begin{abstract}
The aim of the present study is to determine the relationship between the simple motor response and the reaction that occurs when solving a complex motor task. Three groups of 20 experienced persons were studied, respectively, from the gymnastics, judo and football disciplines. The sport reaction was determined by a standard light source test. Using the video computing methodology and the dynamo platform, the time interval $\Delta \mathrm{t} 1$ for the start of the motor action and the time interval $\Delta \mathrm{t} 2$ for the overall realization were determined. A random number generator is used to vary the time interval between the light signals. Stabilization of the mean score at all surveyed individuals was observed at the latest after the seventh trial. From the results of the variation analysis of the three groups of experienced people, the group of judokas, followed by the football players and the gymnasts, stands out with the best achievements. The character of a sport discipline develops in a specific way the speed of the motor response. Standard results could be used to determine the level of some aspects of the sport-technical mastery through biomechanical criteria for realization efficiency.
\end{abstract}

Key words: biomechanical criteria, simple and complex reactions, realization efficiency.

\section{INTRODUCTION}

Sports science distinguishes several relatively independent manifestations of the concept of speed in motion. In a number of sporting disciplines, especially in combats, the speed of motor reaction is essential for the end result. (1-3), In laboratory conditions, simple and complex response rates are usually investigated, which are largely determined by the conductivity and reactivity of the neuromuscular processes. Another meaning has the concept of motor response, when solving tasks with more or less complex muscular synergy is involved. Consequently, the motor response in a particular sport discipline depends directly on the motion control parameters. (4-7). Here we can distinguish reactions with varying degrees of complexity of the motor component depending

\footnotetext{
*Correspondence to: Nikolina Dimitrova,National Sports Academy, Sofia, Department for Foreign Languages and Information Technologies, Studentski grad 1700 Sofia, Bulgaria, E-mail ninansa@abv.bg
}

on the number and nature of the participating muscle groups. $(8,9)$ For that reason, it is appropriate to distinguish the types of actions according to their character - local, regional and global.

The main objective of this study is to determine the relationship between the simple motor response and the reaction that occurs when solving a complex motor task.

\section{METHODS}

Three groups of 20 experienced persons were studied, respectively, from the gymnastics, judo and football disciplines. The sport response was determined by a standard light source test. Since the results in this case also depend on random parameters, the test was repeated several times to stabilize the mean values.

For a comparative analysis of the groups, a motion test was carried out to maximally move the common center of gravity (respectively, the supporting area) $30 \mathrm{~cm}$ to the left or to the right, depending on the direction indicated by 
the light source. Using the video computing methodology and the dynamographic platform, the time interval $\Delta \mathrm{t} 1$ for the start of the motor action and the time interval $\Delta \mathrm{t} 2$ for the overall realization were determined.

\section{RESULTS AND ANALYSIS}

Since the quantification of motor reaction speed depends on a number of randomities and conditions which are uncontrollable by the experimenter, it was necessary to determine in advance the number of the measurements
DIMIROVA N.

required to ensure the accuracy of the assessment. Figure 1 shows the data from a similar experiment. A random number generator is used to vary the time interval between the light signals. In all surveyed persons, stabilization of the mean score was observed at the latest after the seventh trial. Therefore, for the subsequent analysis, we adopted for individual evaluations the average response rates obtained after ten attempts.

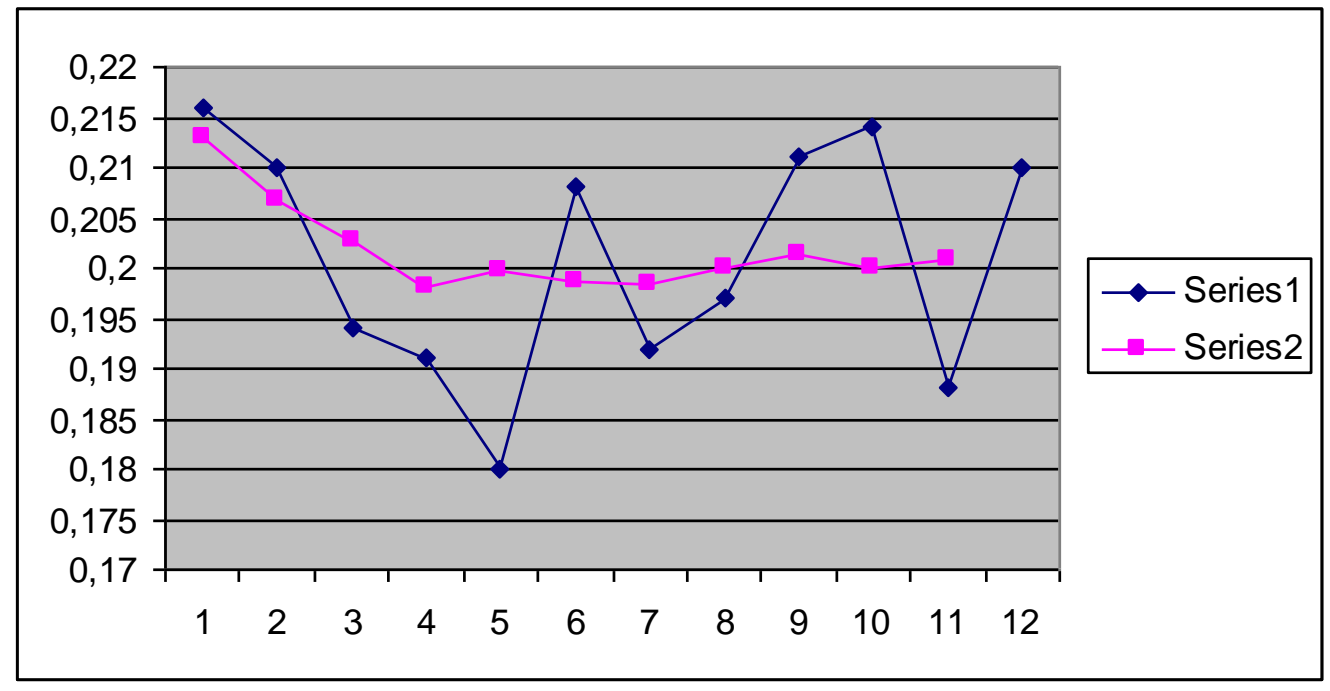

Figure 1. Speed of motor reaction

Table 1 presents the results of the variation analysis of the three groups of surveyed persons from the simple and complex light stimulus tests. In both cases, the group of judokas, followed by footballers and gymnasts, stands out with the best achievements.

Table 1. Results of the variation analysis of the three groups

\begin{tabular}{|l|l|l|l|l|l|l|}
\hline $\begin{array}{l}\text { Characteristics } \\
\text { Group }\end{array}$ & $\Delta \mathrm{t} 1$ & $\mathrm{~S}$ & $\mathrm{~V} \%$ & $\Delta \mathrm{t} 1$ & $\mathrm{~S}$ & $\mathrm{~V} \%$ \\
\hline Gymnastics & 224 & 16 & 7,143 & 316 & 22 & 6,962 \\
\hline Judo & 201 & 8 & 3,98 & 286 & 12 & 4,196 \\
\hline Football & 210 & 24 & 11,43 & 324 & 38 & 11,73 \\
\hline
\end{tabular}

Table 2 presents the data from the simple and complex motion response experiments in the global shift of the CCG. Here the picture changes and there is no statistically significant difference between the groups of the football players and the judokas. What proved to be interesting is the revelation of a possible dependence between the four tests performed a standard simple and complex light source reaction and a simple and complex response to a global shift of the common center of gravity.

Table 2. Simple and complex motion response experiments in the global shift of the CCG.

\begin{tabular}{|l|l|l|l|l|l|l|}
\hline $\begin{array}{l}\text { Characteristics } \\
\text { Group }\end{array}$ & $\Delta \mathrm{t} 1$ & $\mathrm{~S}$ & $\mathrm{~V} \%$ & $\Delta \mathrm{t} 1$ & $\mathrm{~S}$ & $\mathrm{~V} \%$ \\
\hline Gymnastics & 466 & 52 & 11,16 & 516 & 68 & 13,18 \\
\hline Judo & 419 & 21 & 5,012 & 474 & 34 & 7,173 \\
\hline Football & 422 & 38 & 9,005 & 459 & 41 & 8,932 \\
\hline
\end{tabular}


Table 3 presents the results of the correlation analysis performed on the groups of football players and gymnasts. Weaker correlation relationships for the group of gymnasts are to be noticed. On the other hand, it cannot be claimed that there are statistically significant values for the football players too. The Judokas Group demonstrated intermediate values in this respect, with the correlation values obtained between the four tests not exceeding $r=0.54$. The interpretation of this fact can be developed in two directions - the tests measure different parameters of the motor response or sporttechnical skills within the groups is at a low level, which hides the objectively existing relationships. The latter refers primarily to the tests involving more complex intramuscular coordination (Test 3 and 4). The relatively low correlation between Test 1 and 2 clearly demonstrates an objective difference between the assessed response characteristics.

Table 3. Correlation analysis performed on the groups of football players and gymnasts.

\begin{tabular}{|c|c|c|c|c|c|}
\hline № & 1 & 2 & 3 & 4 & \\
\hline 1 & 1 & 0,68 & 0,56 & 0,48 & \multirow{4}{*}{$\begin{array}{l}\bar{\Xi} \\
\text { है } \\
\overline{0} \\
\text { I }\end{array}$} \\
\hline 2 & 0,48 & 1 & 0,48 & 0,42 & \\
\hline 3 & 0,41 & 0,33 & 1 & 0,56 & \\
\hline 4 & 0,32 & 0,38 & 0,33 & 1 & \\
\hline & \multicolumn{4}{|c|}{ Gymnastics } & \\
\hline
\end{tabular}

\section{CONCLUSIONS}

From the results obtained and their analysis, some important conclusions and recommendations could be made.

1. The character of a sport discipline develops in a specific way the speed of the motor response. Hence, the learning process should seek methods and means for its purposeful refinement, and not to rely solely on developing it indirectly through the specifics of motor tasks.

2. The absence of the expected strong correlation relationships between different tests to assess the rapidity of the response indicates the need for developing performance-specific tests for quantitative assessment.

3. Standard results for a simple and a complex response could be successfully used to determine the level of some aspects in sport-technical skills through the biomechanical criteria for realization efficiency.

\section{REFERENCES}

1. Donskoy, D.D., Zatsiorsky, V.M. Biomechanics: Textbook for institutes of physical culture. - Moscow: Fizkul'tura i sport, P. 264, 1979 (In Russian)

2. Ivanov,Sv. (1989) Theory and methods of biomemechanical analysis in the sport field. Dissertation work, Sofia, (In Bulgarian)
3. Winter, D. A. 1997, Biomechanics and motor control of movement, $2^{\text {nd }}$ ed., Wiley and sons, N.Y.,.

4. Adrian, M. J., J. M. Cooper. Biomechanics of human movement. Madison, WI \&:Brown \& Benchmark, 1995.

5. Frank, J., M. Earl. Coordination of posture and movement, Phys. Therapy, v.70, 1990.

6. Yordanov, S., K. Naydenova - Meaning, role and place of the football game for the physical training of the Bulgarian Army soldiers, First International Conference of the Football and Tennis Department, Sofia, 2005, pp. 87-93.

7. Hristakieva, T., E. Mileva. Using Interactive Techniques in English Language Education at the NSA "V. Levski, Sb .: International Scientific and Practical Conference "Current Problems of Physical Culture", 17-18 March 2017, Sofia, NSA PRESS, p.34-39.тура“", 17-18 March 2017 г., София, НСА ПРЕС, с.34-39.

8. Nikolova A, Dimitrova D.; Morphological characteristics of judo cadets with respect to sex-related differences and athletic achievements; Biomedical Human Kinetics,10, pp.169-177, 2018 ;DOI: 10.1515/bhk-2018-0025

9. Albert M., S. Filkova, K. Mollova. Modern tendencies of physical education and sport. University Ed. "St. Kliment Ohridski ", Eighth International Scientific Conference, pp. 70-75. Sofia, 2016 (In Bulgarian) 